Proceedings of the

International Geometry Center

Vol. 13, no. 1 (2020) pp. 49-60

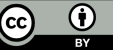

\title{
On functional moduli of surface flows
}

\author{
V. Kruglov, O. Pochinka, G. Talanova
}

\begin{abstract}
Currently, a complete topological classification has been obtained with respect to the topological equivalence of Morse-Smale flows, $[9,7]$, as well as their generalizations of $\Omega$-stable flows on closed surfaces, [4]. Some results on topological conjugacy classification for such systems are also known. In particular, the coincidence of the classes of topological equivalence and conjugacy of gradient-like flows (Morse-Smale flows without periodic orbits) was established in [3]. In the classical paper [8], it was proved that in the presence of connections (coincidence of saddle separatrices), the topological equivalence class of a $\Omega$-stable flow splits into a continuum of topological conjugacy classes (has moduli). Obviously, each periodic orbit also generates at least one modulus associated with the period of that orbit. In the present work, it was established that the presence of a cell in a flow bounded by two limit cycles leads to the existence of an infinitely many stability moduli. In addition, a criterion for the topological conjugation of flows on such cells was found.
\end{abstract}

Анотація. На сьогоднішній день отримана повна класифікація класів топологічної еквівалентності потоків Морса-Смейла, $[9,7]$, а також їх узагальнень - $\Omega$-стійкіх потоків на замкнутих поверхнях, [4]. Відомі деякі результати з класифікації таких систем відносно топологічної спряженості. Зокрема, в роботі [3] встановлено збіг класів топологічної еквівалентності та спряженості для градієнтно-подібних потоків (потоків Морса-Смейла без періодичних орбіт). У класичній праці [8] доведено, що за наявності зв'язки (збігу сідлових сепаратріси), клас топологічної еквівалентності $\Omega$-стійкого потоку розпадається на континуум класів топологічної спряженості (має модулі). Очевидно, що кожна періодична орбіта також породжує як мінімум один модуль, пов'язаний з періодом

The reported study was funded by RFBR according to the research project № 18-3100022 except for Lemma 4.1, which was supported by Laboratory of Dynamical Systems and Applications NRU HSE of the Ministry of science and higher education of the RF grant ag. No 075-15-2019-1931

2010 Mathematics Subject Classification: 37D15, 37D05

UDC УДК 517.938.5

Keywords: non-singular flow, Morse-Smale flow, conjugacy, equivalence, limit cycle DOI: http://dx.doi.org/10.15673/tmgc.v13i1.1714 
орбіти. У даній роботі встановлено, що наявність у потоку області (циліндра), обмеженої двома граничними циклами призводить до існування нескінченного числа модулів стійкості. Також знайдено критерій топологічної спряженості потоків на таких циліндрів.

\begin{abstract}
Аннотация. На сегодняшний день исчерпывающим образом получена топологическая классификация относительно топологической эквивалентности потоков Морса-Смейла [9, 7], а также их обобщений - $\Omega$ устойчивых потоков на замкнутых поверхностях, [4]. Известны некоторые результаты по классификации таких систем относительно топологической сопряженности. Так в работе [3] установлено совпадение классов топологической эквивалентности и сопряженности градиентно-подобных потоков (потоков Морса-Смейла без периодических орбит). В классической работе [8] доказано, что при наличии связки (совпадение седловых сепаратрис), класс топологической эквивалентности $\Omega$-устойчивого потока распадается на континуум классов топологической сопряженности (имеет модули). Очевидно, что каждая периодическая орбита также порождает как минимум один модуль, связанный с периодом орбиты. В настоящей работе установлено, что наличие у потока ячейки, ограниченной двумя предельными циклами приводит к существованию бесконечного числа модулей устойчивости. Кроме того, найден критерий топологической сопряженности потоков на таких ячейках.
\end{abstract}

\title{
1. INTRODUCTION
}

Two flows $f^{t}, f^{\prime t}: M \rightarrow M$ are called topologically equivalent if there exists a homeomorphism $h: M \rightarrow M$ sending trajectories of $f^{t}$ into trajectories of $f^{\prime t}$ preserving orientations of the trajectories. Also, two flows are topologically conjugate whenever there exists a homeomorphism $h: M \rightarrow M$ such that $h \circ f^{t}=f^{\prime t} \circ h$, which means that $h$ sends trajectories into trajectories preserving not only directions but in addition the time of moving.

Finding invariants describing the class of topological equivalence or topological conjugacy of each flow from some class of flows means constructing a topological classification for that class of flows. Note that for some classes their classifications in the sense of equivalence and conjugacy coincide; for other classes these classifications are completely different.

In the present paper we study the problem of topological conjugacy.

Morse-Smale flows were initially introduced and studied on the plane in the classical paper [1] by A. A. Andronov and L. S. Pontryagin. The nonwandering sets of such flows consist of finitely many hyperbolic fixed points and finitely many hyperbolic limit cycles, and besides saddle separatrices intersect only transversally (which means that saddle points of a flow on the plane can not be connected by a separatrix). 
During the twentieth century, there were obtained topological classifications of this important class of flows on different manifolds. The most important combinatorial invariants are the Leontovich-Maier's scheme [5, 6] for flows on the plane, the Peixoto's directed graph [9] for Morse-Smale flows on closed surfaces and the Oshemkov-Sharko's three-colour graph [7] also for Morse-Smale flows on closed surfaces.

All these invariants classify flows only in sense of topological equivalence. The next step is conjugacy. In the paper [3] it was proved that for gradient-like flows (i.e. Morse-Smale flows without limit cycles) the classes of topological equivalence and topological conjugacy on surfaces coincide. But any limit cycle generates infinite many conjugacy classes for each equivalence class (even two cycles with different periods cannot be conjugate). For two saddles connected by a separatrix the invariant (called modulus of stability or modulus of topological conjugacy) was found by J. Palis [8].

The moduli of topological conjugacy have relations to the asymptotic behaviour of the partial time averages [12]. They express the existence of the physical measure of an orbit. Such a measure describes the probability of finding a point of the orbit $x(t)$, for big values of $t$, in the different regions of the phase space [11]. This makes very important to consider two non-singular flows on an annulus bounded by two limit cycles and to find a necessary condition of their topological conjugacy.

In this work we show that any such flow has infinitely many moduli of topological conjugacy.

While considering flows on an annulus it is important to mention recent results on Morse flows on surfaces with a boundary [10].

\section{DyNAMiCS OF THE NON-SINGULAR FLOWS ON AN ANNULUS BOUNDED BY LIMIT CYCLES}

A flow is called non-singular if it has no fixed points and its non-wandering set consists of hyperbolic limit cycles. Every such flow on a surface does not have cycles homotopic to zero because there is no foliations without singularities on a disk.

Let us introduce a class $G$ of non-singular flows $f^{t}$ on an annulus $K=$ $S^{1} \times[0,1]$ such that connected components of $\partial K$ are the two limit cycles $c_{1}$ and $c_{2}, c_{1}=S^{1} \times\{1\}$ is the stable one, $c_{2}=S^{1} \times\{0\}$ is the unstable one, and besides, $c_{1}$ and $c_{2}$ are the only limit cycles of $f^{t}$.

For definiteness we will assume that for any flow $f^{t} \in G$ the orientation of the annulus $K$ coincides on $c_{1}$ with a moving of a point $x \in S^{1} \times\{1\}$ by means $f^{t}$ from $t=0$ to $t=T_{1}$. Say that $c_{1}$ and $c_{2}$ are consistently (inconsistently) oriented if the orientation of $K$ coincides (does not coincide) on $c_{2}$ with a moving of a point $x \in S^{1} \times\{0\}$ by means $f^{t}$ from $t=0$ to 
$t=T_{2}$, see Figure 2.1. We associate with $f^{t}$ a value $\nu=+1(\nu=-1)$ in case of the consistent (inconsistent) orientation respectively.

So, let $f^{t}: K \rightarrow K$ be a flow from $G$, see Figure 2.1, and $T_{1}$ and $T_{2}$ be the periods of $c_{1}$ and $c_{2}$ respectively.

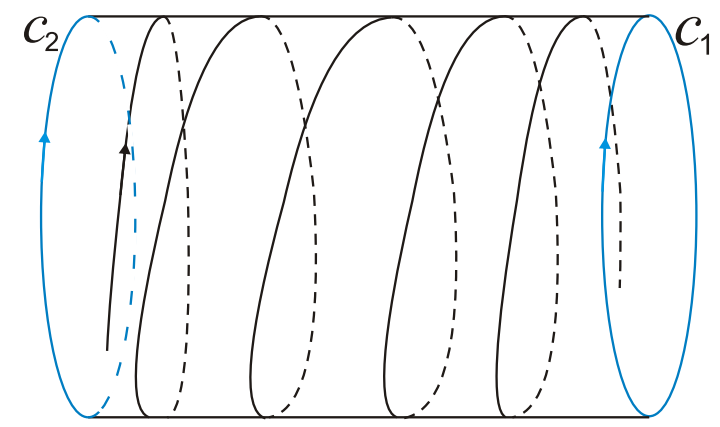

Figure 2.1. An example of a flow from $G$ with the consistent cycle orientation, $\nu=+1$

\section{LiNEARIZATION IN THE NEIGHBORHOOD OF A PERIODIC ORBIT}

Let

$$
\begin{array}{ll}
P_{1}=\left\{\left(x_{1}, y_{1}\right) \in \mathbb{R}^{2}: x_{1} \leqslant 0\right\}, & O x_{1}^{-}=\left\{\left(x_{1}, y_{1}\right) \in P_{1}: y_{1}=0\right\}, \\
P_{2}=\left\{\left(x_{2}, y_{2}\right) \in \mathbb{R}^{2}: x_{2} \geqslant 0\right\}, & O x_{2}^{+}=\left\{\left(x_{2}, y_{2}\right) \in P_{2}: y_{2}=0\right\} .
\end{array}
$$

We define flows $a_{i}^{t}$ by the following system of ODE:

$$
\left\{\begin{array}{l}
\dot{x}_{i}=0 \\
\dot{y}_{i}=1
\end{array}\right.
$$

For $i=1,2$ consider a homeomorphism $g_{i}: P_{i} \rightarrow P_{i}$ given by the formula

$$
g_{i}\left(x_{i}, y_{i}\right)=\left(2^{(-1)^{i}} x_{i}, y_{i}-T_{i}\right)
$$

and the group $G_{i}=\left\{g_{i}^{n} \mid n \in \mathbb{Z}\right\}$. Denote by $\Pi_{i}$ a space orbit of the action of the group $G_{i}$ on $P_{i}$ and by $q_{i}: P_{i} \rightarrow \Pi_{i}$ the natural projection. Then $\Pi_{i}$ is a cylinder and the flow $a_{i}^{t}$ induces via $q_{i}$ a unique flow $\tilde{a}_{i}^{t}$ on $\Pi_{i}$ with a unique limit cycle $\tilde{c}_{i}=q_{i}\left(O y_{i}\right)$, see Figure 3.1.

Let $K_{1}=K \backslash c_{2}$ and $K_{2}=K \backslash c_{1}$. Then by M. Irwin [2] there is a homeomorphism $\eta_{i}: K_{i} \rightarrow \Pi_{i}$ conjugating $\left.f^{t}\right|_{K_{i}}$ with $\tilde{a}_{i}^{t}$.

The existence of a linearizing neighborhood for a periodic orbit is an analogue of the Grobman-Hartmann theorem for a hyperbolic equilibrium point. The linearization generates invariant foliations in a neighborhood of the hyperbolic equilibrium point which depend on a choice of the linearizing 

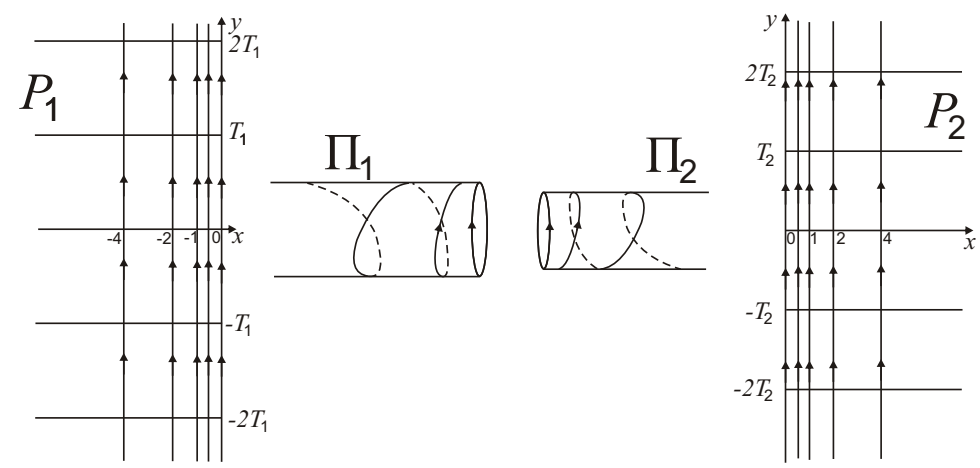

Figure 3.1. $P_{1}, \Pi_{1}, \Pi_{2}, P_{2}$ and the flows on them defined by (3.1)

homeomorphism. A significant difference from the Irwin's linearization is the uniqueness of the linearizing foliation, which we prove in the following section.

\section{UNIQUENESS OF INVARIANT FOLIATION IN A NEIGHBORHOOD OF THE HYPERBOLIC PERIODIC ORBIT}

Lemma 4.1. There exists a unique one-dimensional foliation $\Xi_{i}$ in $K_{i}$ such that its leaves $\xi_{i}$ are cross-sections for the trajectories of flow $f^{t}$ in $K_{i}, f^{T_{i}}(z) \in \xi_{i}$, and $f^{t}(z) \notin \xi_{i}$ for $0<t<T_{i}$ whenever $z \in \xi_{i}$.

Proof. For definiteness we will prove our lemma for $i=1$. The case $i=2$ is similar.

First notice that the existence of at least one foliation $\Xi_{1}$ with the required properties follows from linearization. Indeed, let $\chi_{1}=\left\{y_{1}=c, c \in\right.$ $\mathbb{R}\}$ be a foliation on $P_{1}$, whose leaves are the horizontal semi-lines, and $\tilde{\chi}_{1}=q_{1}\left(\chi_{1}\right)$. Then

$$
\Xi_{1}=\eta_{1}^{-1}\left(\tilde{\chi}_{1}\right)
$$

Let us prove the uniqueness.

Suppose that $\Xi_{1}$ and $\hat{\Xi}_{1}$ are two foliations with the required properties. Let us show that there exists a homeomorphism $\hat{\eta}_{1}: K_{1} \rightarrow \Pi_{1}$ which conjugates $\left.f^{t}\right|_{K_{1}}$ with $\tilde{a}_{1}^{t}$ and $\hat{\eta}_{1}\left(\hat{\Xi}_{1}\right)=\tilde{\chi}_{1}$. Take a segment $\left[z_{0}, f^{T_{1}}\left(z_{0}\right)\right] \subset \hat{\xi}_{1} \in \hat{\Xi}_{1}$ and let

$$
\hat{\eta}_{1}\left(\left[z_{0}, f^{T_{1}}\left(z_{0}\right)\right]\right)=q_{1}([(-2,0),(-1,0)]) .
$$

Both these segments intersect all trajectories of Int $K_{1}$ and Int $\Pi_{1}$. Put $\hat{\eta}_{1}\left(f^{t}(z)\right)=\tilde{a}_{1}^{t}\left(\hat{\eta}_{1}(z)\right)$ for $z \in\left[z_{0}, f^{T_{1}}\left(z_{0}\right)\right]$. Then by continuity such a homeomorphism can be extended to the cycles. 
It follows that the homeomorphism $\tilde{\theta}_{1}=\hat{\eta}_{1} \eta_{1}^{-1}: \Pi_{1} \rightarrow \Pi_{1}$ conjugates the flow $\tilde{a}_{1}^{t}$ with itself. Thus there is a lifting

$$
\theta_{1}=\left(\varphi_{1}\left(x_{1}, y_{1}\right), \tilde{\psi}_{1}\left(x_{1}, y_{1}\right)\right): P_{1} \rightarrow P_{1}
$$

of $\tilde{\theta}_{1}$ (i.e. a map satisfying $q_{1} \circ \theta_{1}=\tilde{\theta}_{1} \circ q_{1}$ ) which conjugates the flow $a_{1}^{t}$ with itself, where $\varphi_{1}\left(x_{1}, y_{1}\right), \tilde{\psi}_{1}\left(x_{1}, y_{1}\right)$ are certain continuous maps.

Notice that the homeomorphism $\theta_{1}$ sends trajectories onto trajectories. Since the trajectories of $a_{1}^{t}$ are vertical lines, it follows that

$$
\varphi_{1}\left(x_{1}, y_{1}\right)=\varphi_{1}\left(x_{1}\right) \text {. }
$$

By definition the flow $a_{1}^{t}$ is given by

$$
a_{1}^{t}\left(x_{1}, y_{1}\right)=\left(x_{1}, y_{1}+t\right)
$$

Hence, by conjugation,

$$
\tilde{\psi}_{1}\left(x_{1}, y_{1}+t\right)=\tilde{\psi}_{1}\left(x_{1}, y_{1}\right)+t .
$$

Put $\psi_{1}\left(x_{1}, y_{1}\right):=\tilde{\psi}_{1}\left(x_{1}, y_{1}\right)-y_{1}$, so $\tilde{\psi}_{1}\left(x_{1}, y_{1}\right)=y_{1}+\psi_{1}\left(x_{1}, y_{1}\right)$. Then we get from (4.1) that

$$
y_{1}+t+\psi_{1}\left(x_{1}, y_{1}+t\right)=y_{1}+\psi_{1}\left(x_{1}, y_{1}\right)+t
$$

whence,

$$
\psi_{1}\left(x_{1}, y_{1}+t\right)=\psi_{1}\left(x_{1}, y_{1}\right), t \in \mathbb{R} .
$$

This means that the map $\psi$ does not depend on $y_{1}$, so

$$
\psi_{1}\left(x_{1}, y_{1}\right)=\psi_{1}\left(x_{1}\right)
$$

Then $\theta_{1}$ has a form

$$
\theta_{1}\left(x_{1}, y_{1}\right)=\left(\varphi_{1}\left(x_{1}\right), y_{1}+\psi_{1}\left(x_{1}\right)\right)
$$

As $\Pi_{1}=P_{1} / G_{1}$, where $G_{1}$ is the cyclic group generated by the homeomorphism $g_{1}\left(x_{1}, y_{1}\right)=\left(x_{1} / 2, y_{1}-T_{1}\right)$, and $\theta_{1}$ is projected from $P_{1}$ to $\Pi_{1}$, it follows that $g_{1} \circ \theta_{1}=\theta_{1} \circ g_{1}$. Therefore

$$
\left(\varphi_{1}\left(x_{1} / 2\right), y_{1}+\psi_{1}\left(x_{1} / 2\right)-T_{1}\right)=\left(\varphi_{1}\left(x_{1}\right) / 2, y_{1}+\psi_{1}\left(x_{1}\right)-T_{1}\right),
$$

whence,

$$
\psi_{1}\left(x_{1} / 2\right)=\psi_{1}\left(x_{1}\right) .
$$

Let us show that in such case $\psi_{1}\left(x_{1}\right)$ is a constant. Indeed, let $x_{1} \in O x_{1}^{-}$ and $y_{1}^{n}=\psi_{1}\left(x_{1} / 2^{n}\right), n \in \mathbb{N}$. As $\psi_{1}\left(x_{1} / 2\right)=\psi_{1}\left(x_{1}\right)$, we get that $y_{1}^{n}=\psi_{1}\left(x_{1}\right)$ for every natural $n$. Moreover, since $\psi_{1}$ is a continuous map, it follows that the constant sequence $\psi_{1}\left(x_{1} / 2^{n}\right)$ converges to $\psi_{1}(0)$ and $\psi_{1}\left(x_{1}\right)=\psi_{1}(0)$ for every $x_{1} \in O x_{1}^{-}$.

Thus $\theta_{1}\left(x_{1}, y_{1}\right)=\left(\varphi_{1}\left(x_{1}\right), y_{1}+b_{1}\right)$, where $b_{1}$ is a constant. Hence, $\theta_{1}$ preserves the foliation $\chi_{1}$. Therefore

$$
\tilde{\chi}_{1}=\tilde{\theta}_{1}\left(\tilde{\chi}_{1}\right)=\hat{\eta}_{1} \eta_{1}^{-1}\left(\tilde{\chi}_{1}\right)=\hat{\eta}_{1}\left(\Xi_{1}\right)
$$


and, since $\tilde{\chi}_{1}=\hat{\eta}_{1}\left(\Xi_{1}\right)$, we obtain that $\Xi_{1}=\hat{\eta}_{1}^{-1}\left(\tilde{\chi}_{1}\right)=\hat{\Xi}_{1}$.

\section{A functional modulus}

Define the following map $\phi: \Pi_{1} \backslash \tilde{c}_{1} \rightarrow \Pi_{2} \backslash \tilde{c}_{2}$ by

$$
\phi=\eta_{2} \eta_{1}^{-1}
$$

and let

$$
\Phi: P_{1} \backslash O y_{1} \rightarrow P_{2} \backslash O y_{2} \quad \Phi\left(x_{1}, y_{1}\right)=\left(\gamma\left(x_{1}, y_{1}\right), \tilde{\delta}\left(x_{1}, y_{1}\right)\right),
$$

be its lift (i.e. a map satisfying $q_{2} \circ \Phi=\phi \circ q_{1}$ ), where $\gamma\left(x_{1}, y_{1}\right), \tilde{\delta}\left(x_{1}, y_{1}\right)$ are continuous maps.

Recall that the trajectories of $a_{1}^{t}$ and $a_{2}^{t}$ are vertical lines, and, by definition, the homeomorphism $\Phi$ conjugates flow $a_{1}^{t}$ with flow $a_{2}^{t}$ sending trajectories onto trajectories. Therefore, $\gamma\left(x_{1}, y_{1}\right)=\gamma\left(x_{1}\right)$. As before, $a_{i}^{t}\left(x_{i}, y_{i}\right)=\left(x_{i}, y_{i}+t\right)$, whence, by conjugation

$$
\tilde{\delta}\left(x_{1}, y_{1}+t\right)=\tilde{\delta}\left(x_{1}, y_{1}\right)+t .
$$

Denote $\delta\left(x_{1}, y_{1}\right):=\tilde{\delta}\left(x_{1}, y_{1}\right)-y_{1}$, so

$$
\tilde{\delta}\left(x_{1}, y_{1}\right)=y_{1}+\delta\left(x_{1}, y_{1}\right) \text {. }
$$

Then

$$
y_{1}+t+\delta\left(x_{1}, y_{1}+t\right)=y_{1}+\delta\left(x_{1}, y_{1}\right)+t
$$

Hence,

$$
\delta\left(x_{1}, y_{1}+t\right)=\delta\left(x_{1}, y_{1}\right)
$$

and

$$
\delta\left(x_{1}, y_{1}\right)=\delta\left(x_{1}\right)
$$

Thus, $\Phi$ has a form

$$
\Phi\left(x_{1}, y_{1}\right)=\left(\gamma\left(x_{1}\right), y_{1}+\delta\left(x_{1}\right)\right) .
$$

As $\Pi_{i}=P_{i} / G_{i}$, where $G_{i}$ is the cyclic group generated by the diffeomorphism $g_{i}(x, y)=\left(2^{(-1)^{i}} x_{i}, y_{i}-T_{i}\right)$ and $\Phi: P_{1} \backslash O y_{1} \rightarrow P_{2} \backslash O y_{2}$ is projected to $\phi: \Pi_{1} \backslash \tilde{c}_{1} \rightarrow \Pi_{2} \backslash \tilde{c}_{2}$ then either the equality $g_{2} \circ \Phi=\Phi \circ g_{1}$ or $g_{2}^{-1} \circ \Phi=\Phi \circ g_{1}$ holds. These cases correspond to 1) $\nu=+1$ and 2) $\nu=-1$, respectively.

Therefore, in the case 1)

$$
\left(2 \gamma\left(x_{1}\right), y_{1}+\delta\left(x_{1}\right)-T_{2}\right)=\left(\gamma\left(x_{1} / 2\right), y_{1}+\delta\left(x_{1} / 2\right)-T_{1}\right)
$$

whence

$$
\delta\left(x_{1}\right)=\delta\left(x_{1} / 2\right)+T_{2}-T_{1}
$$

In the case 2)

$$
\left(\gamma\left(x_{1}\right) / 2, y_{1}+\delta\left(x_{1}\right)+T_{2}\right)=\left(\gamma\left(x_{1} / 2\right), y_{1}+\delta\left(x_{1} / 2\right)-T_{1}\right)
$$


and thus

$$
\delta\left(x_{1}\right)=\delta\left(x_{1} / 2\right)-T_{2}-T_{1} .
$$

Indeed, the map $\delta$ measures a deviation of a leave of $\Xi_{1}$ from a leave of $\Xi_{2}$ in the following sense. Let $x_{1} \in O x_{1}^{-}$. Then $\gamma\left(x_{1}\right) \in O x_{2}^{+}$and $\eta_{2}^{-1}\left(q_{2}\left(\gamma\left(x_{1}\right), 0\right)\right)$ belong to the leaf $\xi_{2} \in \Xi_{2}$ for every $x_{1} \in O x_{1}^{-}$. It follows from the definition of $\delta$ that

$$
f^{\delta\left(x_{1}\right)}\left(\eta_{2}^{-1}\left(q_{2}\left(\gamma\left(x_{1}\right), 0\right)\right)\right)
$$

belongs to the leave $\xi_{1} \in \Xi_{1}$ for every $x \in O x_{1}^{-}$, see Figure 5.1.

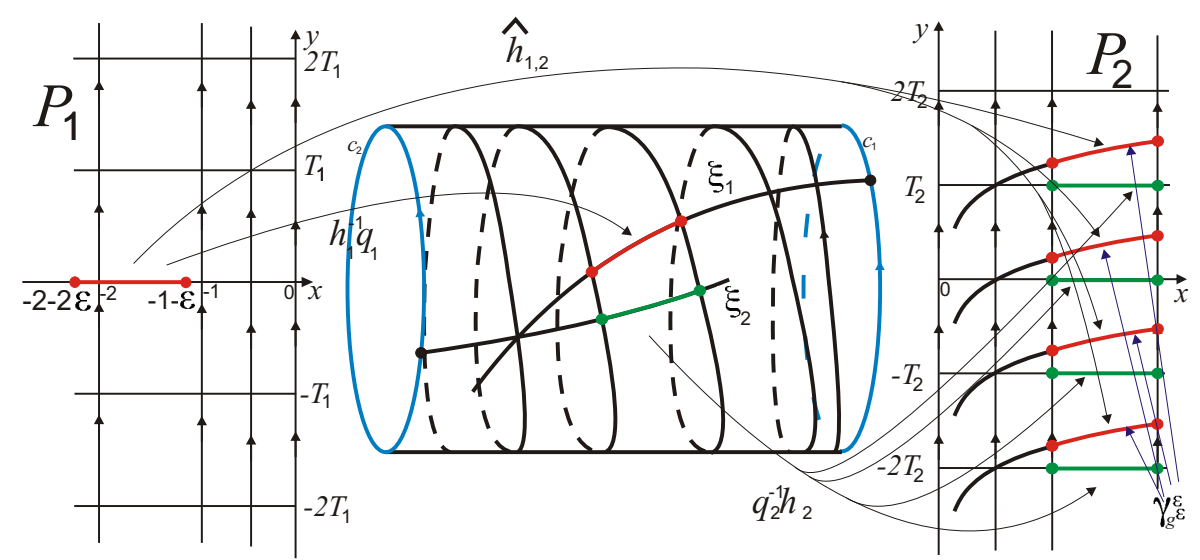

Figure 5.1. Geometrical meaning of $\delta$

The map $\delta: O x_{1}^{-} \rightarrow O y_{2}$ can be projected to $\hat{\delta}: S^{1} \rightarrow S^{1}$ as follows. Let $\beta: O x_{1}^{-} \rightarrow O x_{1}^{-}$be given by the formula $\beta\left(x_{1}\right)=x_{1} / 2$ and

$$
B=\left\{\beta^{n}\left(x_{1}\right) \mid n \in \mathbb{Z}\right\} .
$$

Then $S^{1}=O x_{1}^{-} / B$. Denote by $p_{\beta}: O x_{1}^{-} \rightarrow S^{1}$ the natural projection and let $e: O y_{2} \rightarrow O y_{2}$ be given by the following formulas:

1) for $\nu=+1, e\left(y_{2}\right)=y_{2}+\left(T_{2}-T_{1}\right)$ if $T_{2} \neq T_{1}$, and $e\left(y_{2}\right)=y_{2}+1$ if $T_{2}=T_{1}$

2) for $\nu=-1, e\left(y_{2}\right)=y_{2}-\left(T_{2}+T_{1}\right)$.

Let $E=\left\{e^{n}\left(y_{2}\right) \mid n \in \mathbb{Z}\right\}$. Then $S^{1}=\mathbb{R} / E$. Denote by $p_{e}: O y_{2} \rightarrow S^{1}$ the natural projection. Then we have a well-defined map $\hat{\delta}: S^{1} \rightarrow S^{1}$ given by the formula

$$
\hat{\delta}(s)=p_{e}\left(\delta\left(p_{\beta}^{-1}(s)\right)\right), s \in S^{1} .
$$

We will prove below that the equivalence class of this map with respect to the following equivalent relation is a complete invariant of a flow 
$f^{t}: K \rightarrow K$ up to a topological conjugacy. In other words, such a flow has a functional modulus.

Let us call two maps $\hat{\delta}, \hat{\delta}^{\prime}$ by equivalent if there is a homeomorphism $\hat{\vartheta}: S^{1} \rightarrow S^{1}$ preserving orientation and a constant $s_{0} \in S^{1}$ such that

$$
\hat{\delta}^{\prime}(\hat{\vartheta}(s))=\hat{\delta}(s)+s_{0}
$$

Denote all objects of $f^{\prime t}$ correponding to the ones of $f^{t}$ by the same letters and with additional sign'.

Theorem 5.1. Two flows $f^{t}, f^{\prime t}: K \rightarrow K$ are topologically conjugated iff

1) $T_{i}=T_{i}^{\prime}$;

2) $\nu=\nu^{\prime}$

3) the maps $\hat{\delta}$ and $\hat{\delta}^{\prime}$ are equivalent.

Proof. Necessity. Let $h: K \rightarrow K$ be a homeomorphism conjugating $f^{t}$ with $f^{\prime t}$. Since $\left.h\right|_{c_{i}}, i=1,2$, is also conjugating, it follows that $T_{i}=T_{i}^{\prime}$, which proves 1 ). By assumption for any flow from $G$ the orientation of the annulus $K$ coincides on the stable cycle with a moving in the positive time. This implies that $h$ preserves the orientation of $K$. Then the cycles $c_{1}, c_{2}$ and $c_{1}^{\prime}, c_{2}^{\prime}$ are consistently or inconsistently oriented simultaneously, which establishes 2).

By assumption $h \circ f^{t}=f^{\prime t} \circ h$. Denote by $\theta_{i}: P_{i} \rightarrow P_{i}$ a lift of the homeomorphism $\left.h\right|_{K_{i}}$. Then $\theta_{i}$ topologically conjugates $a_{i}^{t}$ with itself. Similarly to the proof of Lemma 4.1 it is possible to show that

$$
\theta_{i}\left(x_{i}, y_{i}\right)=\left(\varphi_{i}\left(x_{i}\right), y_{i}+b_{i}\right),
$$

where $\varphi_{i}\left(x_{i}\right)$ is a continuous map and $b_{i}$ is a constant.

Notice that we have two maps from $P_{1}$ to $P_{2}$ given by the formulas

$$
\Phi\left(x_{1}, y_{1}\right)=\left(\gamma\left(x_{1}\right), y_{1}+\delta\left(x_{1}\right)\right), \quad \Phi^{\prime}\left(x_{1}, y_{1}\right)=\left(\gamma^{\prime}\left(x_{1}\right), y_{1}+\delta^{\prime}\left(x_{1}\right)\right),
$$

where $\gamma\left(x_{1}\right), \delta\left(x_{1}\right), \gamma^{\prime}\left(x_{1}\right), \delta^{\prime}\left(x_{1}\right)$ are certain continuous maps. On the other hand, we have that $\Phi^{\prime}=\theta_{2} \circ \Phi \circ \theta_{1}^{-1}$ which implies that

$$
\Phi^{\prime}\left(x_{1}, y_{1}\right)=\left(\varphi_{2}\left(\gamma\left(\varphi_{1}^{-1}\left(x_{1}\right)\right)\right), y_{1}+b_{2}+\delta\left(\varphi_{1}^{-1}\left(x_{1}\right)\right)-b_{1}\right) \text {. }
$$

Thus,

$$
\delta^{\prime}\left(x_{1}\right)=\delta\left(\varphi_{1}^{-1}\left(x_{1}\right)\right)+y_{2}^{0},
$$

where $y_{2}^{0}=b_{2}-b_{1}$ or, equivalently,

$$
\delta^{\prime}\left(\varphi_{1}\left(x_{1}\right)\right)=\delta\left(x_{1}\right)+y_{2}^{0} .
$$

Let $\vartheta=\varphi_{1}: O x_{1}^{-} \rightarrow O x_{1}^{-}$and $\hat{\vartheta}=p_{\beta} \circ \vartheta \circ p_{\beta}^{-1}: S^{1} \rightarrow S^{1}$. Then

$$
\hat{\delta}^{\prime}(\hat{\vartheta}(s))=\hat{\delta}(s)+s_{0},
$$

where $s_{0}=p_{e}\left(y_{2}^{0}\right)$ and $s=p_{\beta}\left(x_{1}\right)$. This proves 3$)$. 
Sufficiency. Suppose conditions 1)-3) holds. We should construct a conjugating homeomorphism $h: K \rightarrow K$ for $f^{t}$ and $f^{\prime t}$, and that will prove the Theorem.

By assumption, the cycles $c_{1}, c_{2}$ and $c_{1}^{\prime}, c_{2}^{\prime}$ have the same periods respectively and the same consistent or inconsistent orientations.

Also, there is a preserving orientation homeomorphism $\hat{\vartheta}: S^{1} \rightarrow S^{1}$ and a constant $s_{0} \in S^{1}$ such that

$$
\hat{\delta}^{\prime}(\hat{\vartheta}(s))=\hat{\delta}(s)+s_{0}, s \in S^{1} .
$$

Let $\vartheta: O x_{1}^{-} \rightarrow O x_{1}^{-}$be a lift of $\hat{\vartheta}$, and $\theta_{1}: P_{1} \rightarrow P_{1}$ a homeomorphism defined by the formula

$$
\theta_{1}\left(x_{1}, y_{1}\right)=\left(\vartheta\left(x_{1}\right), y_{1}\right)
$$

Then $\theta_{1}$ conjugates $a_{1}^{t}$ with itself and can be projected to a homeomorphism $h: K_{1} \rightarrow K_{1}$ conjugating $f^{t}$ with $f^{\prime t}$ by the formula

$$
h=\eta_{1}^{-1} \circ q_{1} \circ \theta_{1} \circ q_{1}^{-1} \circ \eta_{1} .
$$

To prove that the homeomorphism $h$ can be extended to $c_{2}$ by conjugating $\left.f^{t}\right|_{c_{2}}$ with $\left.f^{\prime t}\right|_{c_{2}^{\prime}}$ it is enough to show that its lift $\theta_{2}: P_{2} \backslash O y_{2} \rightarrow P_{2} \backslash O y_{2}$ has a form

$$
\theta_{2}\left(x_{2}, y_{2}\right)=\left(\varphi_{2}\left(x_{2}\right), y_{2}+b_{2}\right)
$$

where $\varphi_{2}\left(x_{2}\right)$ is a continuous map and $b_{2}$ is a constant.

Indeed, let $\delta, \delta^{\prime}: \mathbb{R}^{-} \rightarrow \mathbb{R}$ be lifts of $\hat{\delta}$ and $\hat{\delta}^{\prime}$ respectively, such that

$$
\delta^{\prime}\left(\vartheta\left(x_{1}\right)\right)=\delta\left(x_{1}\right)+y_{2}^{0},
$$

where $p_{e}\left(y_{2}^{0}\right)=s_{0}$. By definition of the maps $\Phi$ and $\Phi^{\prime}$ we have that $\theta_{2}=\Phi^{\prime} \circ \theta_{1} \circ \Phi^{-1}$, whence

$$
\theta_{2}\left(x_{2}, y_{2}\right)=\left(\gamma^{\prime}\left(\vartheta\left(\gamma^{-1}\left(x_{2}\right)\right)\right), y_{2}-\delta\left(\gamma^{-1}\left(x_{2}\right)\right)+\delta^{\prime}\left(\vartheta\left(\gamma^{-1}\left(x_{2}\right)\right)\right)\right) .
$$

Put

$$
\varphi_{2}=\gamma^{\prime} \vartheta \gamma^{-1}, \quad x_{1}=\gamma^{-1}\left(x_{2}\right), \quad b_{2}=y_{2}^{0} .
$$

Then $\theta_{2}\left(x_{2}, y_{2}\right)=\left(\varphi_{2}\left(x_{2}\right), y_{2}+b_{2}\right)$.

\section{ReAlization of the invariants By a FLOW}

By Theorem 5.1, a class of the topological conjugacy of a flow $f^{t}: K \rightarrow$ $K$ is uniquely determined by a collection $\left(T_{1}, T_{2}, \nu, \hat{\delta}\right)$, where $T_{1}, T_{2}>0$, $\nu \in\{-1,+1\}$, and $\hat{\delta}: S^{1} \rightarrow S^{1}$ is a continuous map (of arbitrary degree). In this section we will prove here a realization theorem. 
Theorem 6.1. For arbitrary collection $\left(T_{1}, T_{2}, \nu, \hat{\delta}\right)$ with above properties there exists a flow $f^{t} \in G$ from the conjugate class corresponding to these invariants.

Proof. Let $T_{1}, T_{2}>0$ and $\hat{\delta}: S^{1} \rightarrow S^{1}$ be a continuous function. Define a map $\delta: O x_{1}^{-} \rightarrow O y_{2}$ by the formulas

$$
\delta\left(x_{1}\right)=p_{e}^{-1} \circ \hat{\delta} \circ p_{\beta}\left(x_{1}\right), \quad \delta(-1)=0 .
$$

Let $\gamma: O x_{1}^{-} \rightarrow O x_{2}^{+}$given by the formula $\gamma\left(x_{1}\right)=-\frac{1}{x_{1}}$ for $\nu=+1$ and $\gamma\left(x_{1}\right)=-x_{1}$ for $\nu=-1$. Define also the map $\Phi: P_{1} \backslash O y_{1} \rightarrow P_{2} \backslash O y_{2}$ by

$$
\Phi\left(x_{1}, y_{1}\right)=\left(\gamma\left(x_{1}\right), y_{1}+\delta\left(x_{1}\right)\right)
$$

and let

$$
\phi=q_{2} \circ \Phi \circ q_{1}^{-1}: \Pi_{1} \backslash \tilde{c}_{1} \rightarrow \Pi_{2} \backslash \tilde{c}_{2} .
$$

Let us represent the annulus $K$ as a factor space $K=\Pi_{1} \cup_{\phi} \Pi_{2}$ and denote by $r: \Pi_{1} \sqcup \Pi_{2} \rightarrow K$ the natural projection. Then the required flow $f^{t}: K \rightarrow K$ coincides with $r \circ \tilde{a}_{1}^{t} \circ r^{-1}$ on $r\left(\Pi_{1}\right)$ and coincides with $r \circ \tilde{a}_{2}^{t} \circ r^{-1}$ on $r\left(\Pi_{2}\right)$.

\section{Conclusion}

In the paper the non-singular flows on an annulus with only two limit cycles on the boundary components of the annulus are considered and the criteria of the topological conjugacy for such flows is obtained. A complete invariant of the conjugacy class is a triple: a pair of the periods, a type of the orientation consistency, and a continuous map $\hat{\delta}: S^{1} \rightarrow S^{1}$. The direct corollary of this criterion is the existence of uncountably many moduli for the considered flows.

The moduli of topological conjugacy are related with the asymptotic behavior of the partial time averages. They express an existence of a physical measure of an orbit describing the probability of finding a point of the orbit $x(t)$, for big values of $t$, in distinct regions of the phase space. This makes the obtained result very important.

\section{REFERENCES}

[1] A. A. Andronov, L. S. Pontryagin. Rough systems. Doklady Akademii nauk SSSR, 14(5):247-250, 1937 (in Russian).

[2] M. C. Irwin. A classification of elementary cycles. Topology, 9:35-47, 1970, doi: $10.1016 / 0040-9383(70) 90047-9$.

[3] V. E. Kruglov. Topological conjugacy of gradient-like flows on surfaces. Dinamicheskie sistemy, 8(36)(1):15-21, 2018.

[4] Vladislav Kruglov, Dmitry Malyshev, Olga Pochinka. Topological classification of $\Omega$ stable flows on surfaces by means of effectively distinguishable multigraphs. Discrete Contin. Dyn. Syst., 38(9):4305-4327, 2018, doi: 10.3934/dcds. 2018188. 
[5] E. A. Leontovich, A. G. Mayer. About trajectories determining qualitative structure of sphere partition into trajectories. Doklady Akademii nauk SSSR, 14(5):251-257, 1937 (in Russian).

[6] E. A. Leontovič, A. G. Mayer. On a scheme determining the topological structure of the separation of trajectories. Dokl. Akad. Nauk SSSR (N.S.), 103:557-560, 1955 (in Russian).

[7] A. A. Oshemkov, V. V. Sharko. On the classification of Morse-Smale flows on two-dimensional manifolds. Mat. Sb., 189(8):93-140, 1998, doi: 10.1070/SM1998v189n08ABEH000341.

[8] J. Palis. A differentiable invariant of topological conjugacies and moduli of stability. In Systèmes dynamiques III - Varsovie, number 51 in Astérisque, pages 335-346. Société mathématique de France, 1978 (in English).

[9] M. M. Peixoto. On the classification of flows on 2-manifolds. In Dynamical systems (Proc. Sympos., Univ. Bahia, Salvador, 1971), pages 389-419, 1973.

[10] O. O. Prishlyak, A. A. Prus. A three-color graph of Morse flow and a compact surface with a boundary. Nelīnünñ Koliv., 22(2):250-261, 2019, doi: 10.12697/acutm.2018.22.22.

[11] David Ruelle. Chaotic evolution and strange attractors. Lezioni Lincee. [Lincei Lectures]. Cambridge University Press, Cambridge, 1989, doi: 10.1017/CB09780511608773. The statistical analysis of time series for deterministic nonlinear systems, Notes prepared and with a foreword by Stefano Isola.

[12] Floris Takens. Heteroclinic attractors: time averages and moduli of topological conjugacy. Bol. Soc. Brasil. Mat. (N.S.), 25(1):107-120, 1994, doi: 10.1007/BF01232938.

Received: January 3, 2020, accepted: February 27, 2020.

V. Kruglov

National Research University Higher School of Economics, Russian FederaTION

Email: kruglovslava21@mail.ru

ORCID: orcid.org/0000-0003-4661-0288

O. Pochinka

National Research University Higher School of Economics, Russian FederaTION

Email: olga-pochinka@yandex.ru

ORCID: orcid.org/0000-0002-6587-5305

G. Talanova

National Research University Higher School of Economics, Russian FederaTION

Email: Glntalanova@gmail.com

ORCID: orcid.org/0000-0002-4743-4055 